POS $\quad$ PROCEEDINGS

\title{
Explaining X-ray variability in blazars
}

\section{lan $\mathbf{M}^{\mathrm{c}} \mathrm{Hardy}$}

School of Physics and Astronomy

University of Southampton

University Road

Southampton

SO17 1BJ

$U K$

E-mail: imheastro.soton.ac.uk

The X-ray variability of blazars has been observed for many years. The X-ray emission mechanism is now reasonably understood, and, following observations such as the lag of the IR emission (ie the synchrotron component) by the $\mathrm{X}$-rays, is usually explained as synchrotron self-Compton emission from the jet. The X-ray emission is observed to vary very rapidly, usually in a nonlinear way. Sometimes large outbursts can be modelled in terms of a shock propagating down the jet but, even if a shock is the correct physical explanation of the event, what is the source of the underlying variations which drive the shock, and also drive the smaller variations in blazars? We may be able to learn something from the studies of the X-ray variability of non-beamed systems such as Seyfert galaxies and X-ray binary systems. In those systems there are a number of observations, such as the linear relationship between rms variability and flux, and the scaling of characteristic timescales with black hole mass and inversely with accretion rate, which have provided some clues as to the source of the variations. In those systems the variations can be explained quite well as originating as accretion rate variations in the accretion disc which propagate inwards and eventually modulate the X-ray emission region. Thus the key point is that the origin of the variations and the source of the X-rays are physically separated. Here I examine the X-ray observations of blazars such as 3C273 and 3C279 and show that they agree very well with the same model which explains the variations in Seyfert galaxies and X-ray binaries. In particular, the characteristic timescale in 3C273 scales very well with characteristic timescales from Seyfert galaxies. There is no need to adjust the timescale to take account of time dilation, thereby implying that the origin of the variations (note - not the source of the X-rays) lies outside the jet. Thus I propose here that X-ray variations which we see from blazars originate in accretion rate fluctuations in the disc which then propagate inwards, modulating any central non-moving X-ray emitting corona, and then carry on and modulate, or possibly produce, the X-ray emission region in the jet.

Workshop on Blazar Variability across the Electromagnetic Spectrum

April 22-25 2008

Palaiseau, France 


\section{Introduction}

There is considerable evidence that the emission from blazars arises in a relativistic jet oriented towards the observer (eg [9]). In the radio through optical bands the emission is usually ascribed to synchrotron emission and in the X- and Gamma-ray bands the emission is agreed to arise from Compton scattering of some seed photon population by the relativistic electrons in the jet that were responsible for the synchrotron emission. In the X-ray band the seed photon population is probably the synchrotron population (ie synchrotron self-Compton emission, SSC) but in the Gamma-ray band, the seed population may be ambient UV photons from the accretion disc (external Compton emission, EC). These mechanisms have been described by many authors, eg see [9] and references therein.

The origin of blazar variability is, however, less well understood. It has generally been described in terms of outbursts or flares, with each flare being thought of as a separate, independent, event. There are many papers in the literature which analyse such flares and attempt to derive the parameters of the underlying emission region (eg [25]). The flares have often been attributed to shocks in the jet and shocked-jet models do provide a good explanation of the main observational facets of large flux variations (e.g. [10]).

There is, however, a deeper question. Even if the emission from the jet does come mainly from shocked regions, where do the original variations, from which the shocks are generated, come from? In this paper I explore the possibility that the original variations arise outside of the jet and that the jet can be thought of merely as a transfer function (probably non-linear) which converts the external variations into observable flux variations.

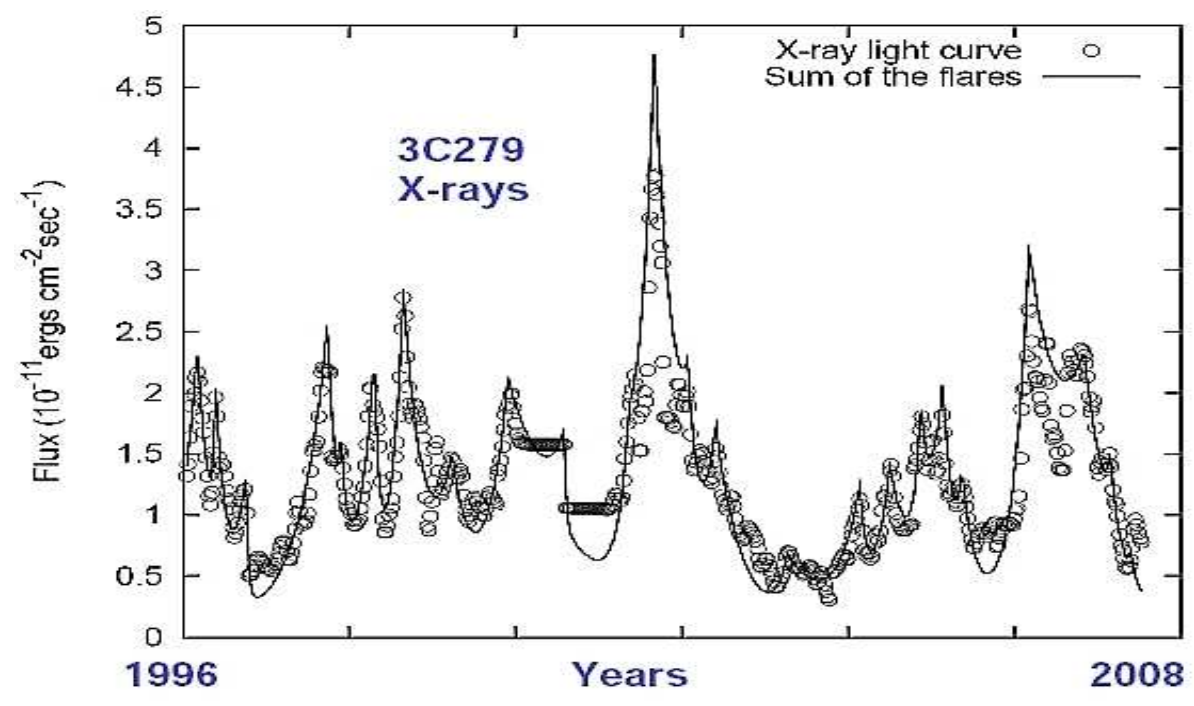

Figure 1: 2-10 keV RXTE lightcurve of 3C279 [2]. Here the lightcurve has been fitted as the sum of a number of outbursts. Whilst such fitting can certainly be done, the question we are addressing here is 'What is the origin of the outbursts?' 


\section{What we can learn from Galactic X-ray Binary Sources and Seyfert Galaxies}

Over the last decade there have been many observations of the X-ray variability of Seyfert galaxies and GBHs with RXTE and considerable effort has been put into modelling these observations. It is now clear that X-ray variability is a red-noise process, with coupling between variations on long and short timescales. We understand how variability timescales scale with mass and accretion rate and we have a model which can explain the origin of the variations. In the following sections I will discuss the main diagnostic observations which have lead us to our present understanding of GBH and Seyfert galaxy variability and I will test the observations of blazars against those diagnostics. In particular I will discuss the rms-flux relationship and the scaling of characteristic timescales. I will also briefly mention the measurement of the lag between the hard and soft X-ray bands as a function of Fourier frequency, although its interpretation is somewhat more complex in the case of blazars. I will concentrate here on the two best observed blazars, ie 3C273 and $3 \mathrm{C} 279$.

\section{The rms-flux relationship}

\subsection{Non-beamed black hole systems}

Following discussions regarding the relative merits of measuring powerspectra in terms of absolute power or rms power, it was found that the rms variability of GBH lightcurves (ie the integral of the PSD over the observable frequency range) varies linearly with flux (Fig.2, left panel, from [22]). It was also found that a linear rms-flux relationship applies to Seyfert galaxies (eg Fig.2, right panel, and [17, 15]).
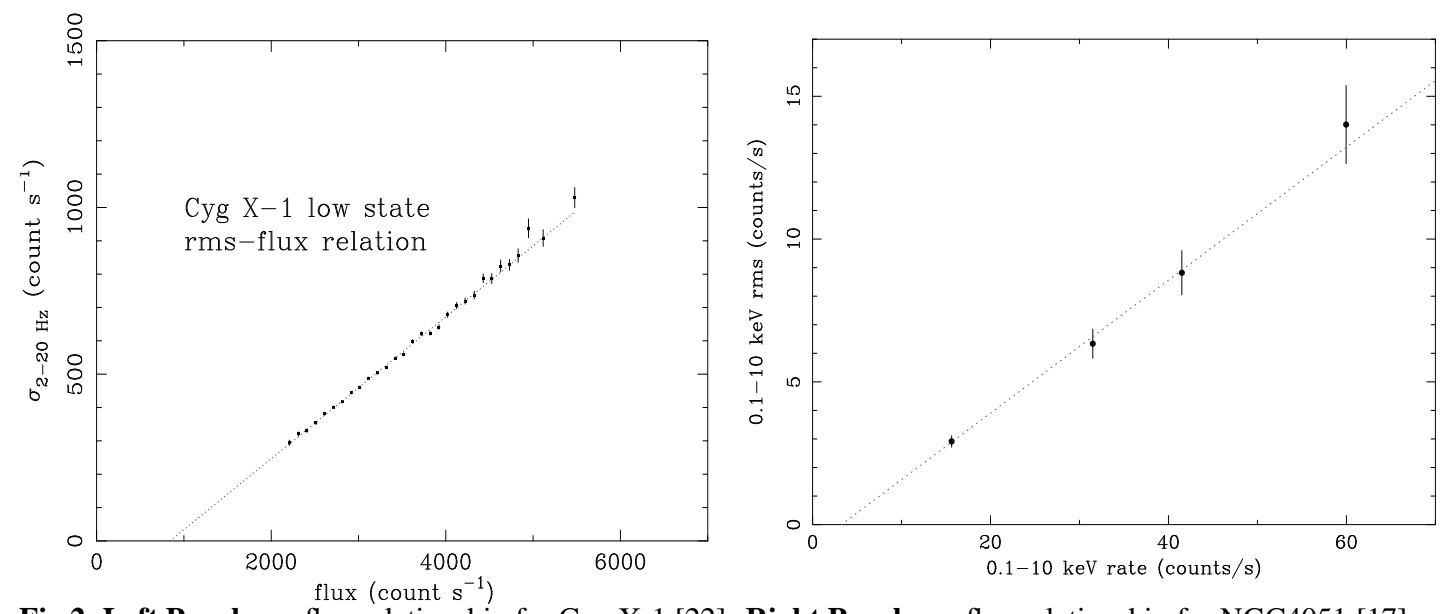

Fig.2. Left Panel rms-flux relationship for Cyg X-1 [22]. Right Panel rms-flux relationship for NGC4051 [17]

The fact that short timescale variations (ie those that determine the rms) decrease in amplitude when the long timescale variations (ie those that determine the mean flux level) decrease means that there must be a link between variations on different timescales. Thus the overall flux variations must be the result of a multiplicative, rather than additive, process. A model which can produce such variations was proposed by Lyubarskii [7]. In this model variations are produced in annuli in 
the accretion disc and propagate inwards where they modulate the emission from annuli further in, thereby producing a linear rms-flux relationship. The longest wavelength which can be produced depends on the radius of the annulus and shorter wavelengths are damped as they travel inwards. Thus we can effectively associate smaller radii with shorter timescales of variability.

The variations travel inwards where they eventually hit the X-ray emitting region, causing variations in the emitted X-ray flux $[6,3,1]$. The critical aspect of this model is that the source of the variations is separated from the source of the X-rays.

We note that the previous popular shot-noise model for the production of variability, where the lightcurve is made up of many independent and randomly timed shots, does not easily produce a linear rms-flux relationship. If all the shots are identical we expect a constant rms.

If a lightcurve was made up of the sum of many different components, then the central limit theorem would lead us to expect a 'normal' or Gaussian distribution of fluxes. However a multiplicative link between components implies that the distribution of fluxes should follow a log-normal distribution [24]. Indeed it is possible to show that if the amplitude of variations on any timescale is multiplied by the amplitude of variations on all longer timescales, then the eventual observed lightcurve will be the exponential of an underlying linear lightcurve, thus again leading us to expect a log-normal flux distribution. In Cyg X-1 such a log-normal flux distribution is indeed seen (Fig.3, from [24]), which strengthens our confidence in the propagating fluctuation model.

The multiplicative relationship between variations on different timescales directly implies that the lightcurves will be non-linear, as can also be seen from the fact that the observed lightcurve can be expressed as the exponential of an underlying linear lightcurve. The sources with the largest fractional rms variability, ie with the greatest modulations of shorter timescale variability (eg Narrow Line Seyfert 1 galaxies, NLS1s) will therefore be the most non-linear, as is observed [24].

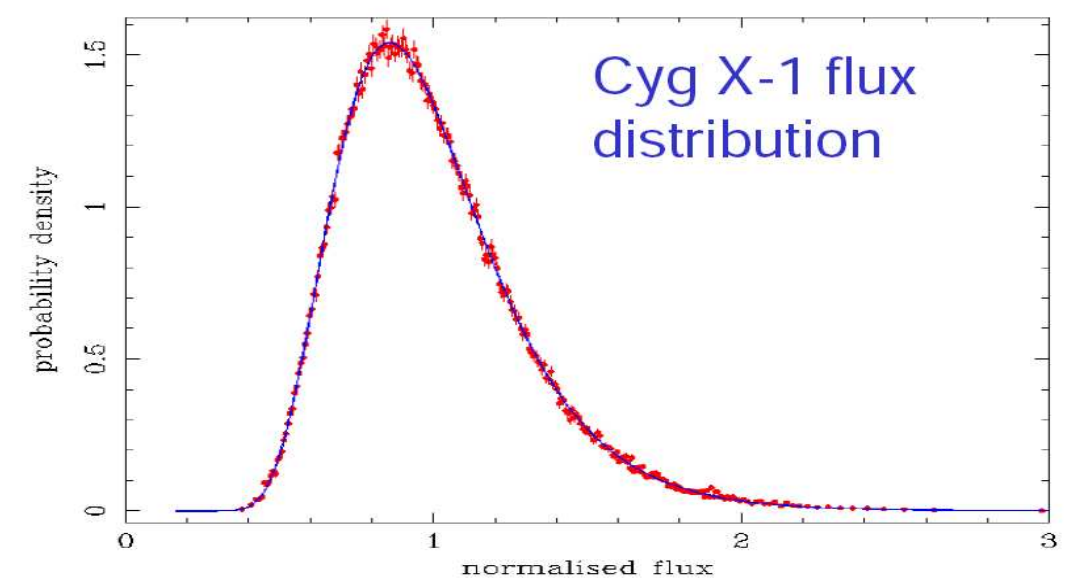

Figure 3: Flux distribution of Cyg X-1 fitted to a log-normal distribution [?, from]]uttley05

\subsection{Blazars}

Here I concentrate, for the moment, on 3C279 (Fig. 1) and 3C273 (Fig. 4). Whilst 3C279 is accepted by all observers to be an archtypal blazar, the credentials of $3 \mathrm{C} 273$ are sometimes ques- 
tioned. 3C273 certainly contains a relativistic jet, and superluminal motion is detected in its radio components [21]. Also the synchrotron (IR) component leads the X-rays [11, 12] by about a day, showing that a substantial fraction of the X-ray emission arises by synchrotron self-Compton emission, which is probably from a jet, rather than by thermal Comptonisation such as is expected in the $\mathrm{X}$-ray emitting non-beamed corona of a Seyfert galaxy. A detailed discussion regarding the relative contribution of the unbeamed X-ray emission in 3C273 has been presented[19] which concludes that the blazar contribution usually dominates, particularly above $2 \mathrm{keV}$. As that is the band which is sampled by RXTE and which we discuss here, we proceed assuming that the majority of the $R X T E$ flux discussed here is of blazar origin.

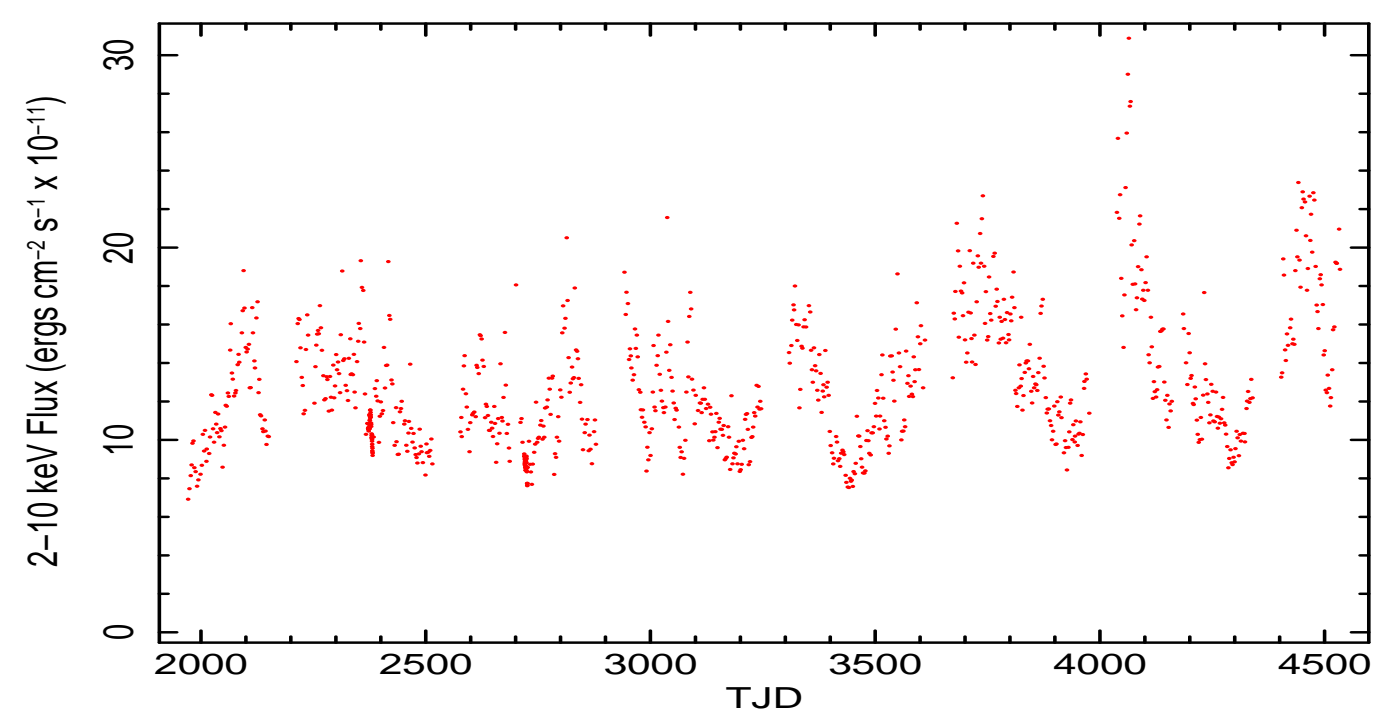

Figure 4: RXTE 2-10 keV X-ray lightcurve of 3C273.

Amongst the blazar community it is often said that 'sources vary more when they are brighter'. That statement is entirely consistent with the rms-flux relationship and so here I have calculated the X-ray rms-flux relationship for the blazars 3C273 and 3C279 (Fig.5). In both cases we note a strong linear relationship, implying that the same basic process is driving the variability in these blazars as in the non-beamed Seyfert galaxies and GBHs. Extrapolation of the fit to zero rms leaves, in both cases, an offset on the flux axis, as is seen in the non-beamed objects. Thus either the linear relationship breaks down at low fluxes and bends gently to the origin (which we are unable to measure), or there is a component of the X-ray emission which does not take part in the variability process. The origin of any constant component is unclear but may arise in an extended jet which is much larger than the variable component. I note that, relative to the peak measured flux, the constant component is larger in $3 \mathrm{C} 273$ than in $3 \mathrm{C} 279$ and may come from the extended 
$\mathrm{X}$-ray jet in 3C273 [4].
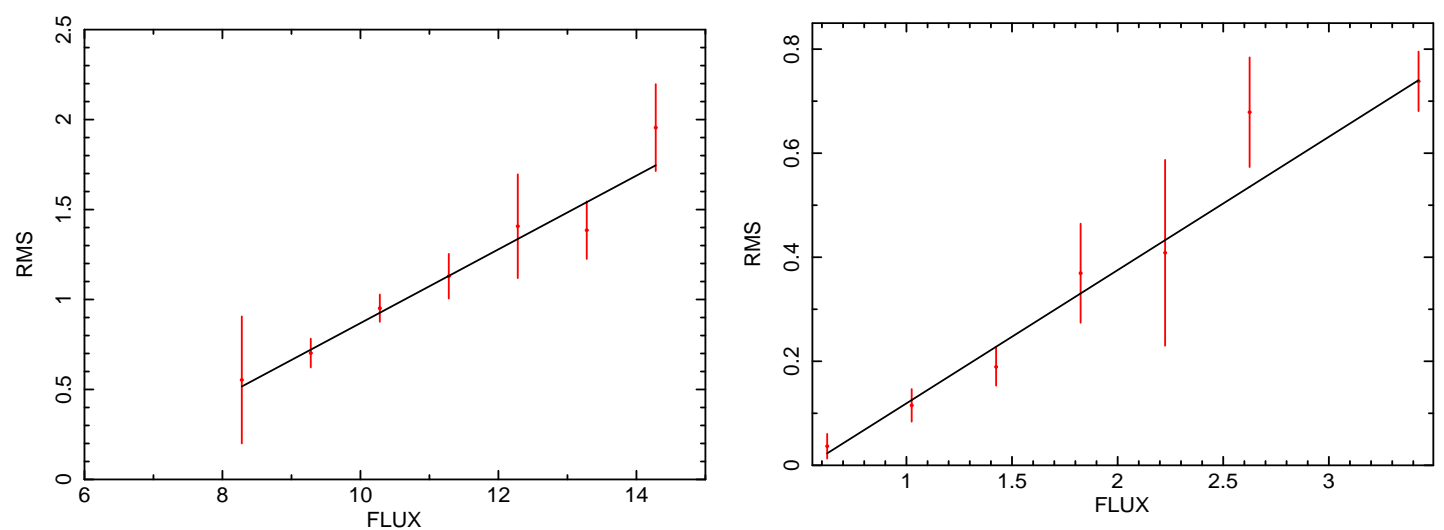

Fig.5. Left Panel rms-flux relationship for 3C273 derived from the X-ray lightcurve shown in Fig.4.

Right Panel rms-flux relationship for 3C279 derived from the lightcurve shown in Fig.1

\section{Powerspectra and Scaling of Characteristic Timescales}

The X-ray powerspectral densities (PSDs) of GBHs and Seyfert galaxies can be described very well as red-noise processes, such as would be produced by the variability process described above. Seyfert galaxies, in particular, have PSDs which, at low frequencies are described by simple powerlaws, ie $P(v) \propto v^{-\alpha}$ with $\alpha \sim 1$. Above a particular frequency, $v_{B}$, or timescale $T_{B}=1 / v_{B}$, the PSD steepens to $\alpha \geq 2$. This timescale may correspond to the viscous timescale associated with the inner edge of the accretion disc and the overall PSD shape is quite consistent with the Lyubarskii/Kotov/Churazov emission model.

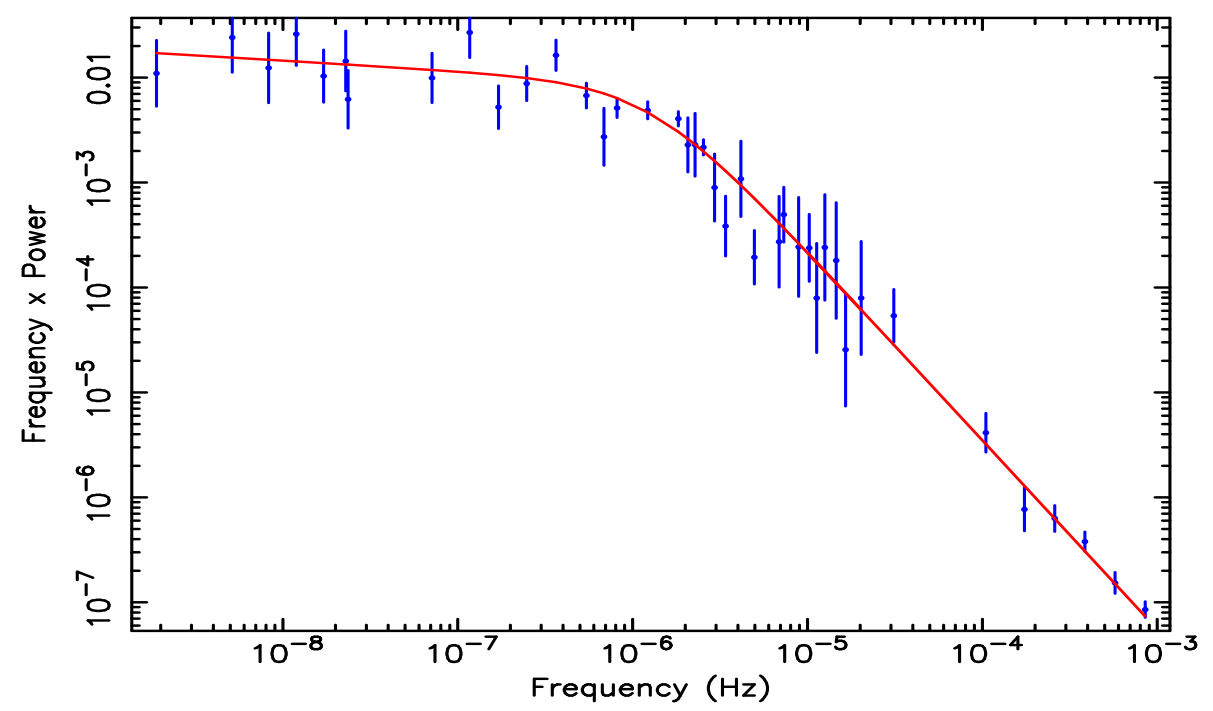

Figure 6: PSD of 3C273, derived from observations by RXTE EXOSAT, and many previous observatories, unfolded from the observational sampling pattern. 
There has long been speculation regarding the scaling of $T_{B}$ with black hole mass $[14,18,23$, 8] but there is a large scatter in $T_{B}$ for any given black hole mass, $M$ [17]. As the high accretion rate NLS1s have the shortest values of $T_{B}$ for any given black hole mass, it was suggested that the scatter may be attributable to variations in accretion rate [17] and a recent detailed analysis [16] shows that, for both Seyfert galaxies and GBHs, $T_{B} \propto M / \dot{m}_{E}$, where $\dot{m}_{E}$ is the accretion rate in Eddington units. It has been shown that this scaling relationship applies to almost all non-beamed accreting objects [5].

We have recently computed the PSD of 3C273, using a combination of RXTE data and data from previous satellites going back to the 1970s. The resultant PSD which, on long timescales, is probably has the best defined of any active galaxy, is shown in Fig. 6. The PSD looks exactly like that of Seyfert galaxies such as MCG-6-30-15 [15] and NGC4051, and Cyg X-1 in the soft state [17]. In Fig. 7 I plot the expected vs. observed values of $T_{B}$ for a sample of AGN and GBHs, using the best-fit scaling relationship between $T_{B} M$ and $\dot{m}_{E}$ from [16] and here I add 3C273. It can be seen that $3 \mathrm{C} 273$ fits the relationship derived for non-beamed black hole systems extremely well.

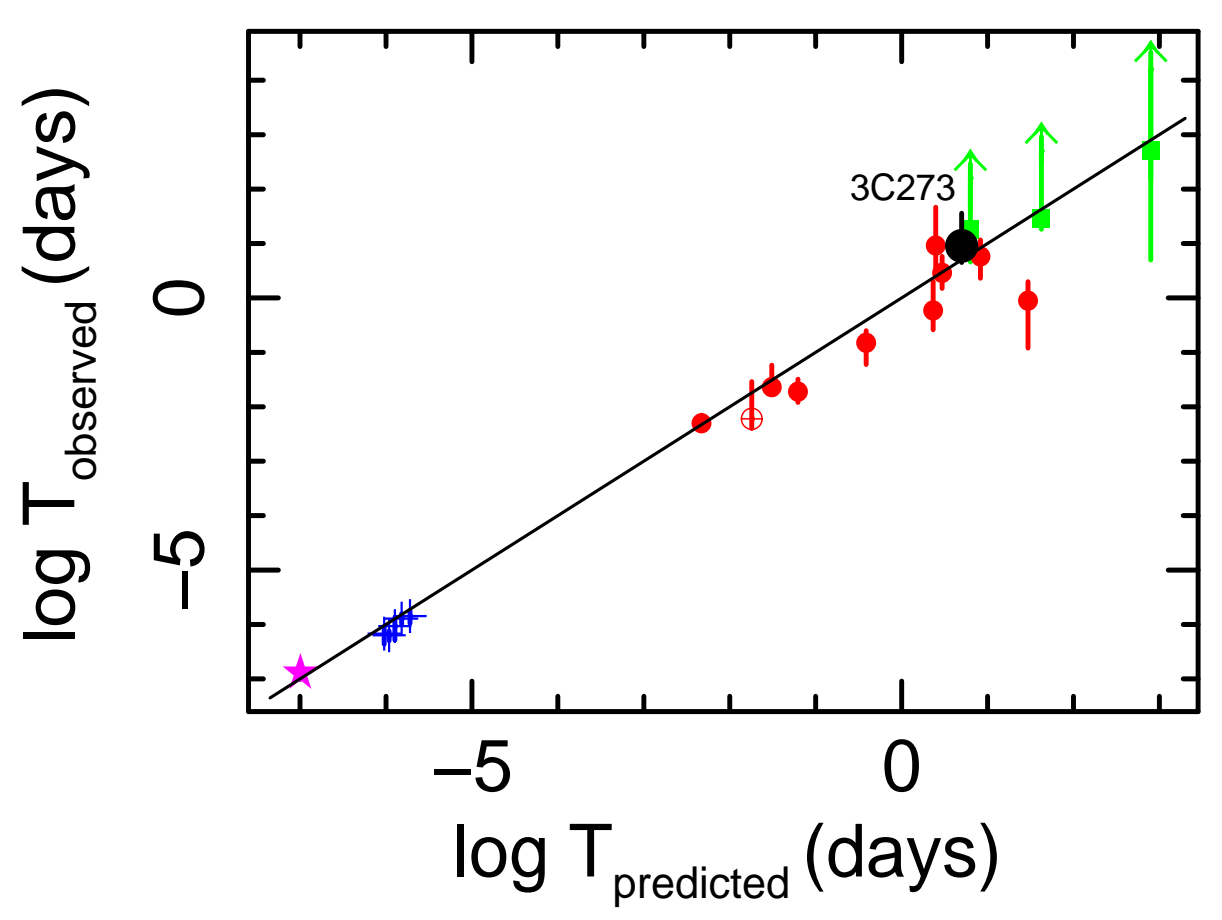

Figure 7: Observed PSD bend timescales, $T_{B}$ compared with the expected bend timescales calculated from the scaling relationship between $T_{B}$ black hole mass $(M)$ and accretion rate $\left(\dot{m}_{E}\right)$ for Seyfert galaxies and soft-state GBHs [16], ie $T_{B} \propto M^{1.12} \dot{m}_{E}^{-0.98}$. The red circles are Seyfert galaxies which were included in the fit and the green squares with upward pointing arrows (ie no good limit on their upper observed bend timescales) are three Seyfert galaxies which were not included in the fit. The blue crosses represent Cyg $\mathrm{X}-1$ and the magenta square is GRS1915+105 in a radio quiet state. 3C273 (black filled circle) has not been included in the fit and is simply plotted here. We can see that 3C273 agrees very well with the fit for the non-beamed black hole systems.

At first sight, it is surprising that $3 \mathrm{C} 273$ fits the relationship for non-beamed black hole systems so well. We might have expected that, as the bulk of the X-ray emission comes from a jet which 
is moving towards us at a relativistic velocity, that the observed timescale might be contracted by relativistic time dilation and so would be shorter than the timescale predicted using the black hole mass and accretion rate. That does not appear to be the case. The implication then is that the clock which is producing the variations (ie the source of the variations) does not lie within the jet, but simply modulates the jet emission. For example, if we were to stand outside the jet and tap on it at a particular frequency, a distant observer measuring that frequency would measure the same frequency as we originally imposed in our rest frame. Only if the clock producing the variations were inside the jet would an external observer measure a time-contracted frequency. The agreement between the scaling relationships for non-beamed black hole systems and for 3C273 thus strengthens the proposition that the variations originate in the accretion disc and then propagate inwards, modulating any non-beamed X-ray emission region, whether over the inner disc or in a central corona, and continue out to modulate the X-ray emission from the jet.

\section{Hard-soft X-ray Lags}

The lag between the hard and soft X-ray emission provides a useful diagnostic of the emission geometry in GBHs and Seyfert galaxies. Although the geometrical interpretation of lags is more difficult in the case of blazars, it is still useful to examine their application to non-beamed X-ray sources as they can, in principle, still provide some diagnostic of the emission geometry.

If the X-ray source has a radial energy profile, e.g. with higher energy photons being preferentially produced closer to the black hole, then in the propagating fluctuation model lags will be expected between the soft and hard X-rays corresponding to the time it takes variations to propagate from the centroid of the soft X-ray emission to that of the hard X-ray emission. Thus the hard $\mathrm{X}$-rays will lag behind the soft X-rays. If we measure the lag as a function of Fourier timescale then as long as the timescale originates from a radius which is well outside all of the X-ray emission region, the lag will remain relatively constant with timescale. However once the timescale corresponds to a radius which is within the X-ray emitting region, then the effective centroid of the soft X-ray emission which can still be affected by perturbations at that timescale, ie the centroid of the soft X-ray emission within that radius, will move closer to the effective centroid of the hard $\mathrm{X}$-ray emission. Thus the lags will decrease. Thus for GBHs and Seyfert galaxies, where the X-ray emission region may either lie above the inner part of the accretion disc or perhaps be in the form of some central corona around the black hole which extends beyond the inner radius of the accretion disc, then a characteristic lag pattern may be expected in the X-ray emission. Just such a pattern is seen in many GBHs and AGN $[17,15,13]$.

There is an additional complication in interpreting observations of Fourier resolved lags (ie lag spectra) in that the emission process itself (eg Comptonisation) may add its own lag to any lag which is produced by propagation time (ie emission geometry) arguments. Indeed some researchers consider that all of the lag may be produced by the details of the emission mechanism but the fact that the shape of the powerspectrum and the shape of the lag spectrum can be simultaneously fitted by the same propagation model (eg Fig 8 from [13]) does strengthen that model. Nonetheless, in standard thermal Comptonisation models, such as are commonly invoked to explain Seyfert and GBH spectra, we expect the hard X-rays to lag behind the soft X-rays as more scatterings are required to raise the seed photons up to the higher energies. This lag is in the opposite sense to 


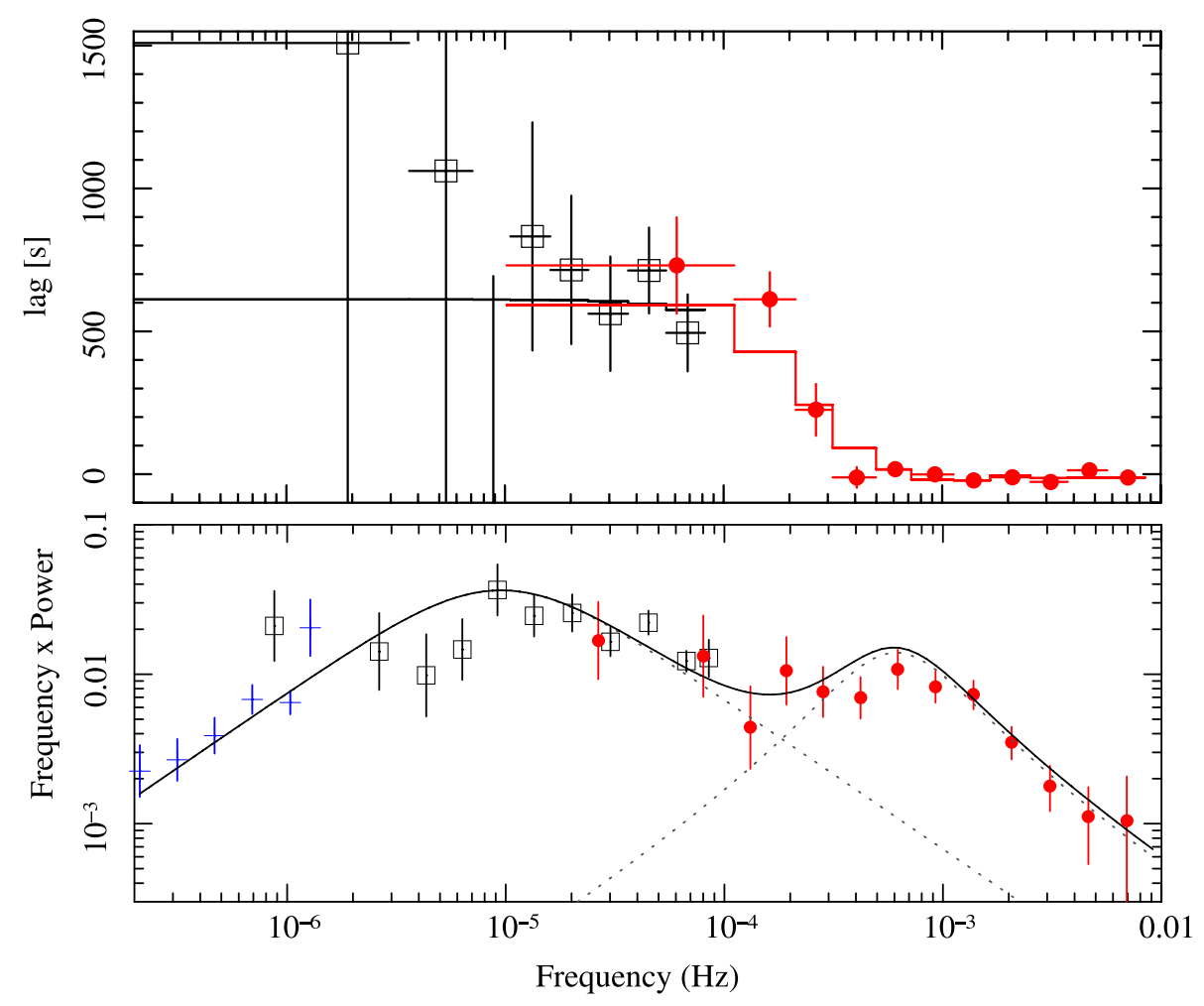

Figure 8: X-ray PSD (bottom panel) and lag between the hard and soft band (top panel) as a function of Fourier frequency for the NLS1 Ark564 [13]. Here a positive lag means that the hard X-ray band lags behind the soft band. The PSD is described by two Lorentzian shaped components, shown as dotted lines. The lag spectrum is relatively constant at both high and low frequencies but note how the rapid change in lag occurs at the frequency at which the PSD changes from one Lorentzian component to another.

that expected from the propagating fluctuation model. It may, however, dominate on short fourier timescales where the propagation lag should be very small. Indeed it is seen that the hard X-rays do lag the soft X-rays in Ark564 on the shortest timescales, although another possible explanation for the soft lag is that the soft $\mathrm{X}$-rays result from reprocessing in the accretion disc.

In the case of blazars the interpretation of lags is likely to be more difficult. If the variability arises largely as a result of perturbations being imposed upon a steadily emitting jet, then the lags may indeed represent the travel time between the hard and soft X-ray emission centroids. As the hard X-ray emission region will lie closer to the black hole, we expect the hard band to lead, but the lag should be constant for all Fourier timescales. The hard band leading is, indeed, what is usually observed, e.g. [20]. Alternatively, if we believe that the X-ray emission arises entirely from discrete events (eg shocks, which may have been triggered by the fluctuations entering the jet from the corona) then again we will expect the hard band to lead, e.g.[10], as the small emission volume containing electrons with an initially hard energy distribution, evolves into a larger volume dominated by lower energy electrons. In this case the lags may be longer for longer Fourier timescales, corresponding to larger emission regions. However detailed modelling is required to produce quantitative predictions. 


\section{A Possible Scenario for Blazar X-ray Variability}

I have shown that there are a number of similarities between the X-ray variability properties of beamed and non-beamed black hole systems. These similarities suggest that the same process is driving the variability in all systems. This process could be fluctuations in the accretion flow through the disc. These fluctuations could modulate any X-ray emission region above the disc, or around the central black hole. The fluctuating accretion flow could then propagate out into the jet where it could produce variable X-ray emission, either by modulating the emission from the 'quiescent' jet or perhaps by generating the emission via shocks (eg [10]). The exact process by which the X-rays might be produced in the jet is still unclear and so it is represented, in the schematic Fig 10 simply by the box labelled 'jet physics; non-linear response'.

I conclude by encourage observers to test the diagnostics which I have described above, and which were derived from observations of non-beamed systems, to blazars, to determine whether the underlying emission process is the same in all accreting objects.

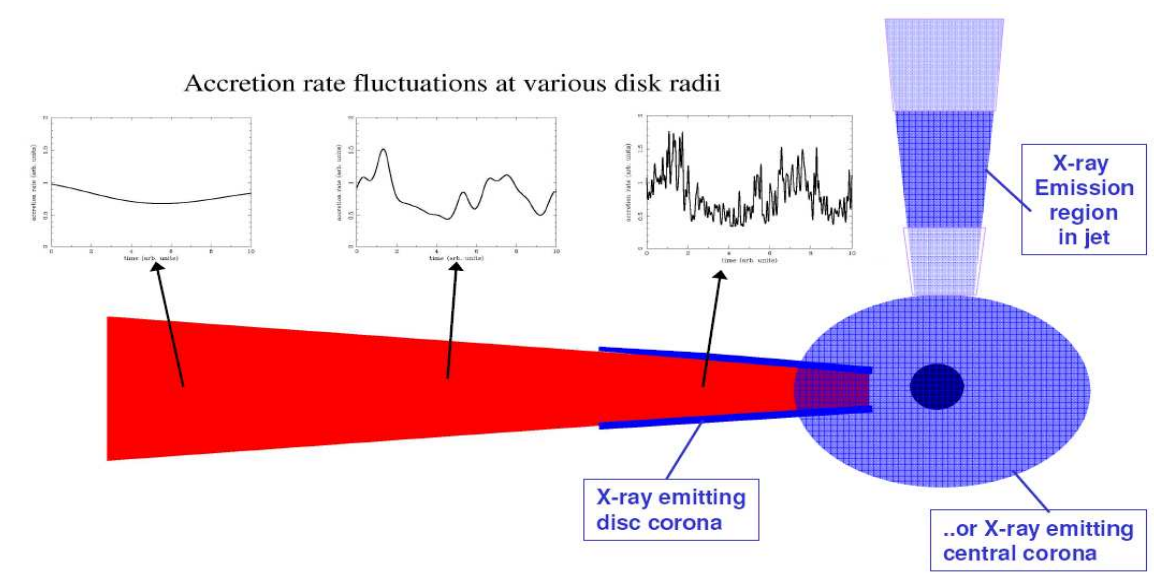

Figure 9: A possible geometric scenario to explain blazar variability. Variations are generated within the accretion disc and propagate inwards to modulate inner X-ray emitting regions and also the X-ray emitting region in the jet.

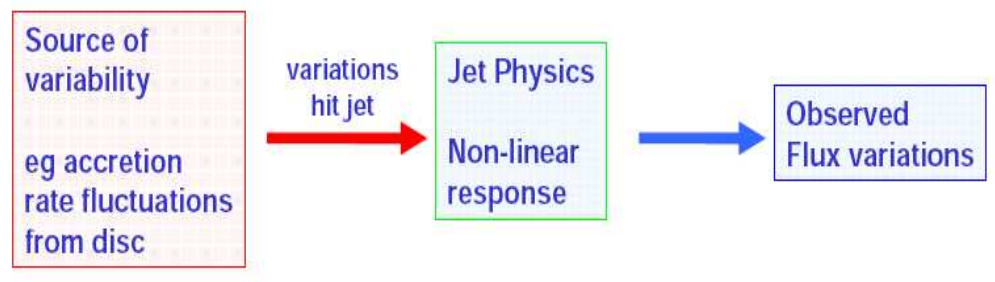

Figure 10: The X-ray emission scenario shown in Fig.9. explained in words. 


\section{References}

[1] Arévalo, P. and Uttley, P., 2006, MNRAS, 367, 801

[2] Chatterjee, R., Jorstad, S. G., Marscher, A. P., Oh, H., McHardy, I. M., Aller, M. F. et al., 2008, ApJ, 689, 79

[3] Churazov E., Gilfanov M., Revnivtsev M., 2001, MNRAS, 321, 759

[4] Jester S., Harris D. E., Marshall H. L., Meisenheimer K., 2006, ApJ, 648, 900

[5] Körding, E. G., Migliari, S., Fender, R., Belloni, T., Knigge, C. and McHardy I.M., 2007, MNRAS, 380,301

[6] Kotov O., Churazov E., Gilfanov M., 2001, MNRAS, 327, 799

[7] Lyubarskii Y. E., 1997, MNRAS, 292, 679

[8] Markowitz A., Edelson R., Vaughan S., Uttley P., George I. M., Griffiths R. E., Kaspi S., Lawrence A., McHardy I., Nandra K., Pounds K., Reeves J., Schurch N., Warwick R., 2003, ApJ, 593, 96

[9] Marscher A. P., these proceedings.

[10] Marscher A. P., Gear W. K., 1985, ApJ, 298, 114

[11] Mㄷardy I., Lawson A., Newsam A., Marscher A., Robson I., Stevens J., 1999, MNRAS, 310,571

[12] Mㄷardy I., Lawson A., Newsam A., Marscher A. P., Sokolov A. S., Urry C. M., Wehrle A. E., 2007, MNRAS, 375, 1521

[13] MHardy I. M., Arévalo, P., Uttley, P., Papadakis, I. E., Summons, D. P., Brinkmann, W. and Page, M. J., 2007, MNRAS, 382, 985

[14] McHardy I. M., 1989, in Hunt J., Battrick B., eds, Two Topics in X-Ray Astronomy, Volume 2. Vol. 296 of ESA Special Publication,X ray variability of active galactic nuclei. pp 1111-1124

[15] Mㄷardy I. M., Gunn K. F., Uttley P., Goad M. R., 2005, MNRAS, 359, 1469

[16] M M Hardy I. M., Koerding E., Knigge C., Uttley P., Fender R. P., 2006, Nature, 444, 730

[17] M McHardy I. M., Papadakis I. E., Uttley P., Page M. J., Mason K. O., 2004, MNRAS, 348, 783

[18] Papadakis, I. E. and $\mathrm{M}^{\mathrm{c}}$ Hardy, I. M., 1995, MNRAS, 273, 923

[19] Pietrini P., Torricelli-Ciamponi G., 2008, A\&A, 479, 365

[20] Takahashi T., Tashiro M., Madejski G., Kubo H., Kamae T., Kataoka J., Kii T., Makino F., Makishima K., Yamasaki N., 1996, ApJL, 470, L89+

[21] Unwin S. C., Cohen M. H., Biretta J. A., Pearson T. J., Seielstad G. A., Walker R. C., Simon R. S., Linfield R. P., 1985, ApJ, 289, 109

[22] Uttley P., McHardy I. M., 2001, MNRAS, 323, L26

[23] Uttley, P., McHardy, I. M. and Papadakis, I. E., 2002, MNRAS, 332, 231.

[24] Uttley P., McHardy I. M., Vaughan S., 2005, MNRAS, 359, 345

[25] Wehrle A. E., Pian E., Urry C. M., Maraschi L., McHardy I. M., Lawson A. J., Ghisellini G., Hartman R. C. etal., 1998, ApJ, 497, 178 